\title{
Joint Motion Control for Lower Limb Rehabilitation Based on Iterative Learning Control (ILC) Algorithm
}

\author{
Wei Guan, ${ }^{1}$ Lan Zhou, ${ }^{1}$ and YouShen Cao $\mathbb{D}^{2}$ \\ ${ }^{1}$ Department of Physical Education, Northwest University, Xi'an 710127, China \\ ${ }^{2}$ College of Arts and Sports, Dong-A University, Busan 49315, Republic of Korea \\ Correspondence should be addressed to YouShen Cao; 1876808@donga.ac.kr
}

Received 30 November 2020; Revised 5 January 2021; Accepted 19 January 2021; Published 28 January 2021

Academic Editor: Wei Wang

Copyright (c) 2021 Wei Guan et al. This is an open access article distributed under the Creative Commons Attribution License, which permits unrestricted use, distribution, and reproduction in any medium, provided the original work is properly cited.

At present, the motion control algorithms of lower limb exoskeleton robots have errors in tracking the desired trajectory of human hip and knee joints, which leads to poor follow-up performance of the human-machine system. Therefore, an iterative learning control algorithm is proposed to track the desired trajectory of human hip and knee joints. In this paper, the experimental platform of lower limb exoskeleton rehabilitation robot is built, and the control system software and hardware design and robot prototype function test are carried out. On this basis, a series of experiments are carried out to verify the rationality of the robot structure and the feasibility of the control method. Firstly, the dynamic model of the lower limb exoskeleton robot is established based on the structure analysis of the human lower limb; secondly, the servo control model of the lower limb exoskeleton robot is established based on the iterative learning control algorithm; finally, the exponential gain closed-loop system is designed by using MATLAB software. The relationship between convergence speed and spectral radius is analyzed, and the expected trajectory of hip joint and knee joint is obtained. The simulation results show that the algorithm can effectively improve the gait tracking accuracy of the lower limb exoskeleton robot and improve the follow-up performance of the human-machine system.

\section{Introduction}

With the coming of the 21 st century, the aging of the population is becoming more and more serious. Some diseases and injuries lead to functional disorders of lower limb movement. Lower limb assisted exoskeleton robot is a wearable human-computer integrated equipment, which can complete various high-intensity tasks through the guidance of human lower limbs [1]. The equipment provides assistance for human walking, enhances human walking ability and speed, and relieves human fatigue under high load and long-time walking. Motion control algorithm is the core part of the control system of exoskeleton robot [2]. The key technology of the control algorithm is to recognize the gait information of the wearer and detect the wearer's motion intention. The research team of Berkeley University of California developed a lower extremity exoskeleton robot (BLEEX) using a hybrid control algorithm of position control and sensitivity amplification control [3]. The exoskeleton robot (hal-5) developed by the University of Tsukuba, Japan, judges the wearer's movement intention by collecting biological current on human skin and controls the exoskeleton machine. The results show that the lower limb rehabilitation exoskeleton will follow the wearer to exercise [4]; the lower limb rehabilitation exoskeleton developed by the State Key Laboratory of Fluid Transmission and Control of Zhejiang University uses the analysis and decisionmaking method of fuzzy logic to identify the wearer's movement intention [5]; the fuzzy adaptive PID (proportion integral) is adopted by Shanghai Jiaotong University. The application of derivative control algorithm to hybrid lower limb exoskeleton robot increases the load capacity of human body.

As we all know, many controlled objects in the actual control system are nonlinear, and the motion process has periodicity, such as robot control system. Although we hope to understand the characteristics of the controlled object clearly in the actual control, so as to obtain the accurate 
mathematical model to realize the accurate motion control, it is difficult to achieve because of the complexity of the system. PID control, which is widely used in industrial field, can control the nonlinear system [6]. However, it often takes a lot of time to adjust PID parameters, and it may not achieve good control effect and control efficiency. The ILC strategy is suitable for the control of such periodic nonlinear systems because of its model independent characteristics and strong self-learning ability, which also lays the foundation for the rapid development of ILC. Miao et al. [7] did a lot of research on various control laws of iterative learning, such as the study of control algorithm theory and application, made a detailed explanation, and made clear the concept of ILC. Since then, research teams around the world have conducted extensive research on ILC, including the design of learning control law, algorithm stability analysis, and robustness analysis of learning control and convergence rate [8]. The research on the theoretical basis of these algorithms provides a good theoretical basis for the application of ILC in various fields. At present, ILC has been widely used in industrial control, such as robot repetitive operation control. Due to the excellent self-learning ability of ILC to the controlled system, it has also been applied to exercise rehabilitation with periodic movement characteristics in recent years. For example, Ghanbari et al. [9] used a phase ahead ILC algorithm in the upper limb rehabilitation based on NMES to reconstruct the motor function of the affected limb. In addition, ILC is also used to control and obtain the desired ankle movement to achieve foot drop correction. The process of iterative learning is to make the trajectory tracking error of the controlled system smaller and smaller by repeatedly running the control system. Through repeated learning of the controlled system, the controller makes the actual trajectory approach the expected trajectory continuously. ILC is characterized by strict mathematical description and low requirements for controlled system. Therefore, ILC is usually suitable for nonlinear systems with uncertain parameters, which is the unique control advantage of ILC.

The Swiss Federal Institute of Technology Zurich has developed a gait rehabilitation training robot LOKOMAT that can be put on the market [10]. The rehabilitation robot has four degrees of freedom and is used with a weight-reducing device and a treadmill to drive patients to reciprocate gait movement. The exoskeleton uses a DC servo motor to drive the lead screw to drive the upper and lower legs to swing in the sagittal plane. In addition, in the follow-up study, the posture control of the exoskeleton stepping on the joint was increased, which can prevent the foot injury caused by the patient's toe drop. At the same time, in order to realize the active and passive rehabilitation training of patients, strategies such as PD control, impedance control, force/position hybrid control, and adaptive control were, respectively, proposed. The experiment proved the effectiveness of the control strategy [11]. At present, although LOKOMAT has been put into use in some world-renowned rehabilitation centers and hospitals, it is still unacceptable for most rehabilitation hospitals due to its high price.
In this paper, based on the poor tracking performance of lower limb exoskeleton robot following human motion, iterative learning control algorithm is suitable for repeated work in limited time and can achieve the desired trajectory tracking. Therefore, based on iterative learning control algorithm, this paper puts forward the following control model of lower limb exoskeleton robot; through the analysis of human lower limb structure, establishes the dynamic model of lower limb exoskeleton robot; uses iterative learning control algorithm to design the motion control system and carries out convergence analysis; and uses MATLAB software to analyze the human hip. Tracking the desired gait trajectory of the joint and knee joint verifies the superiority of the iterative learning control algorithm and shows good application value.

\section{Dynamic Modeling of Lower Limb Exoskeleton Robot}

Biomechanical simulation and experimental studies show that the power consumed by human body in sagittal plane is equivalent to the sum of frontal plane and horizontal plane. From the control point of view, robot belongs to multivariable nonlinear automatic control system, and each control task itself is a dynamic system. Robot dynamics is the basis of robot technology research. Studying robot dynamics is to pave the way for better solving control problems.

2.1. Structural Analysis of Human Lower Limbs. After a lot of simplifications to the dynamics of the musculoskeletal system, the modeling process is still extremely complicated, with many model parameters. The process of musculoskeletal system producing joint motion is highly nonlinear, such as the process of muscle fiber recruitment and joint viscoelasticity. In addition, the time-varying characteristics of the parameters of the musculoskeletal system of the human body are also very obvious, and the related parameters may change in different time periods and different posture measurements [12], and under the action of electrical stimulation, the acceleration of muscle fatigue will also bring about physiological parameters. These factors not only bring great difficulty to the identification of model parameters, but also the accuracy of parameter identification cannot be guaranteed, which brings a lot of challenges to model-based joint motion control. Therefore, in order to obtain a good control effect, the control target of the control system must be the self-learning and adaptive capabilities of the musculoskeletal system.

Figure 1 shows the phase diagram of human gait cycle. For unilateral lower limb, a gait cycle can be divided into two phases: support phase and swing phase. Among them, the support phase accounts for $60 \%$ of the whole movement period, including the single foot support state and the bipedal support state, and the swing phase accounts for $40 \%$ of the whole swing period [13]. Therefore, in the man-machine coupling system, the rotation of the hip joint and knee joint in the single foot support state drives the swing of the thigh, leg, and foot to realize the posture movement of the human 


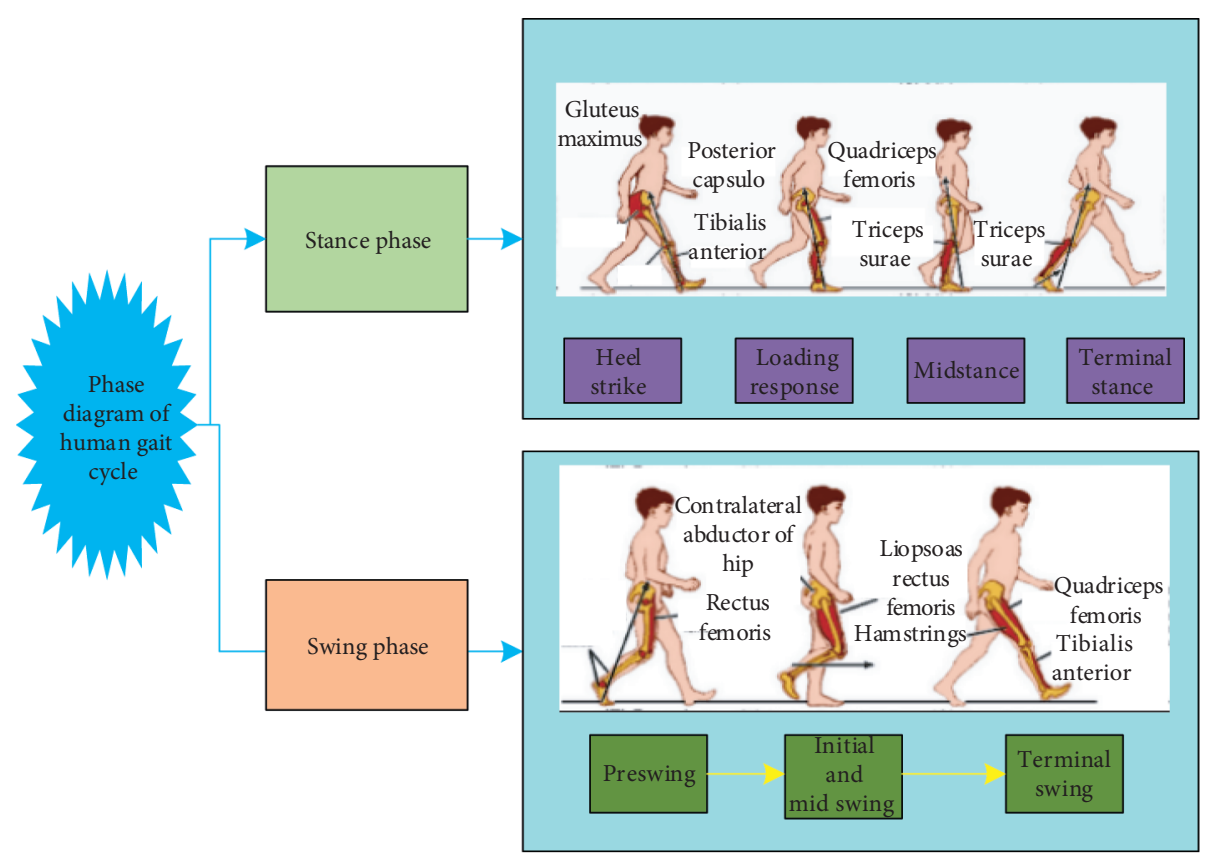

Figure 1: Phase diagram of human gait cycle.

lower limbs. This article attempts to apply the ILC algorithm to the design of the NMES joint motion control system, in order to use the ILC algorithm to achieve stable and accurate joint motion control without relying on the characteristics of the precise model and the powerful self-learning ability.

Therefore, this section first explains the theoretical basis of ILC and then designs an iterative learning control joint motion control system and simulates based on the musculoskeletal system model established in the previous section to verify the feasibility and effectiveness of the algorithm.

According to the normal movement space of human body, the degree of freedom of human lower limb movement includes thigh flexion and extension, internal rotation, external rotation, and adduction and abduction; leg flexion and extension; foot flexion and extension, internal rotation, external rotation, and adduction and abduction. As the main form of human movement, walking completes the body movement through the alternate support of two feet. In the process of human walking, it is stipulated that a complete gait cycle starts from one foot following the ground and ends with another landing on the same side.

2.2. Dynamic Model of Lower Limb Exoskeleton Robot. The dynamic equation of lower limb exoskeleton robot mainly describes the relationship between robot motion and control force. The movement of walking posture mainly occurs in the sagittal plane. A complete support phase can be divided into one-foot support state and two-foot support state. In the stage of single foot support, the driving torque of hip joint and knee joint of swinging leg is larger than that of bipedal support state; because the ankle joint mainly adjusts the direction of human motion in the horizontal plane, the joint driving torque provided in the sagittal plane is smaller [14]. Therefore, the swing leg of lower limb exoskeleton robot can be simplified into the swing leg model as shown in Figure 2 in sagittal plane. Assuming that the mass of the connecting rod is concentrated in the center of the link, the simplified swing leg model of the exoskeleton robot is analyzed. The man-machine system is taken as the research object by the Lagrange method. The kinetic energy and potential energy of the human-machine system are analyzed, and the dynamic equation is obtained:

$$
\tau-T_{a}=A(p) p+B(p, p) p+C(p) .
$$

In equation (1), define the joint angular displacement $p=\left(\begin{array}{l}\theta_{1} \\ \theta_{2}\end{array}\right)$, inertial force matrix $A(p)=\left(\begin{array}{ll}m_{11} & m_{12} \\ m_{21} & m_{22}\end{array}\right)$, and $m_{12}=m_{21}=0.25 a_{2} L_{2}^{2}+0.5 a_{2} L_{1} L_{2} \cos \theta_{2}$,

$m_{11}=0.25 a_{1} L_{1}^{2}+a_{2} L_{1}^{2}+0.25 a_{2} L_{2}^{2}+a_{2} L_{1} L_{2} \cos \theta_{2}$,

$m_{22}=0.25 a_{2} L_{2}^{2}$.

Centrifugal force matrix is

$$
\begin{aligned}
B(p, p) & =\left(\begin{array}{ll}
b_{11} & b_{12} \\
b_{21} & b_{22}
\end{array}\right), \\
b_{11} & =-a_{2} L_{1} L_{2} \sin \theta_{2}, \\
b_{12} & =-0.5 a_{2} L_{1} L_{2} \sin \theta_{2}, \\
b_{21} & =0.5 a_{2} L_{1} L_{2} \sin \theta_{2}, \\
b_{22} & =a_{2} L_{1} L_{2} \sin \theta_{2} \theta_{1} .
\end{aligned}
$$

Define the gravity vector: 


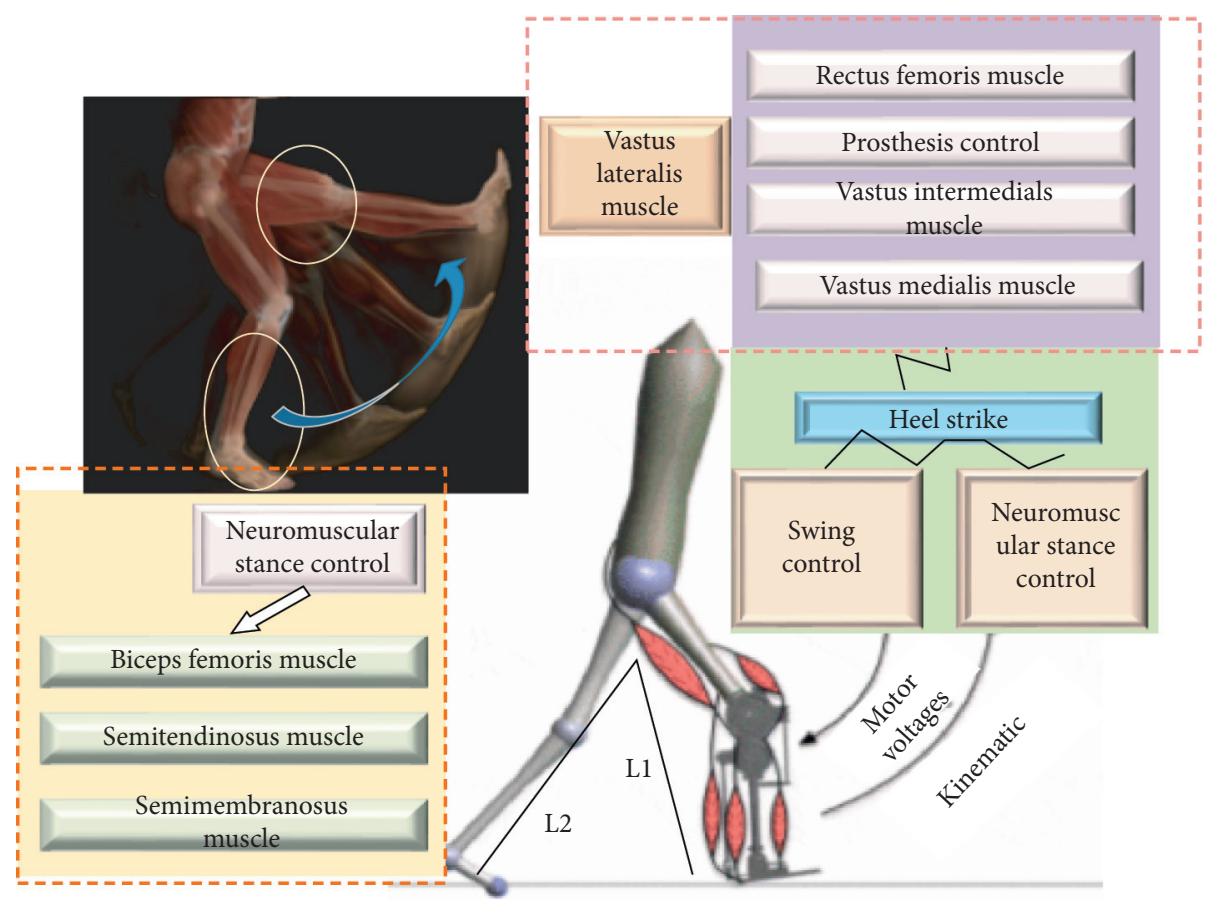

Figure 2: Swing leg model.

$$
\begin{aligned}
C(p)= & \left(\begin{array}{c}
c_{1} \\
c_{2}
\end{array}\right), \\
c_{1}= & 0.5 a_{1} g L_{1} \cos \theta_{1}+a_{2} g L_{1} \cos \theta_{1} \\
& +0.5 a_{2} g L_{2} \cos \left(\theta_{1}+\theta_{2}\right), \\
c_{1}= & 0.5 a_{2} g L_{2} \cos \left(\theta_{1}+\theta_{2}\right) .
\end{aligned}
$$

Among them, $a_{1}$ and $a_{2}$ represent the mass of thigh and lower leg, respectively, $L_{1}$ and $L_{2}$ represent the length of thigh and lower leg in man-machine system, and $\theta_{1}$ and $\theta_{2}$ represent the motion angles of hip joint and knee joint, respectively.

The control system of the lower limb exoskeleton rehabilitation robot involves human-computer interaction. The impact of the patient is in the circuit. In order to consider the safety of the patient and the interference of the patient's exoskeleton control, the control system should have high real-time performance and good robustness. In addition, because the lower extremity exoskeleton rehabilitation robot interacts with the patient and the patient sends out an active movement intention as an input signal for the exoskeleton control, the human-computer interaction control algorithm is indispensable. In order to prevent the affected limb from confronting the exoskeleton due to abnormal muscle activities (such as spasm), interactive control should be able to provide a safe and active and flexible training method [15]. At the same time, in order to encourage patients to actively participate in rehabilitation training, so that patients have a sense of success, interactive control will obtain the patient's active movement intention from sensor signals to achieve active training.

\section{Control of Lower Limb Exoskeleton Robot}

Lower extremity exoskeleton robots assisting patients with gait rehabilitation training have become the mainstream trend of adjuvant therapy for patients with hemiplegia caused by spinal cord injury such as stroke. It can replace part of the therapist, increase training intensity, delay training time, etc. Therefore, how to effectively control the lower extremity exoskeleton rehabilitation robot to assist patients in completing gait rehabilitation training has become a main focus of research at home and abroad. It can be known from the theory of rehabilitation that when the patient is suffering from paresis (pre-rehabilitation), the muscle strength of the patient is very small. Passive rehabilitation is mainly used to improve the muscle tension of the patient and inhibit abnormal muscle movement. In the passive training mode, the lower limb exoskeleton orthosis drives the patient's lower limbs to make a fixed gait movement according to the reference trajectory according to the fitted experimental data, without adjusting the trajectory [16]. However, with the continuous deepening of the rehabilitation training process, the patient's muscle strength continues to improve, and the patient is willing to actively participate in the rehabilitation training. In patientassisted gait rehabilitation training, the gait training trajectory of the exoskeleton robot can be adjusted by the interaction between the patient and the exoskeleton, so that the walking gait is determined jointly by the exoskeleton robot and the patient instead of one rigid gait improving patient participation and sense of success. Therefore, for the above different rehabilitation stages, this section proposes the control algorithms for passive and active auxiliary rehabilitation training for patients. 
Exoskeleton robot has the dynamic characteristics of high nonlinearity, strong coupling, and time-varying. When designing the controller, due to the uncertainty of the mathematical model, the designed controller may lead to the system performance instability. Iterative learning control (ILC) is characterized by simple learning algorithm and independent of the detailed model of the controlled system. It is suitable for the controlled object with repetitive motion in finite time interval. On the basis of establishing the dynamics model of lower limb exoskeleton robot, the tracking error is adjusted to the learning signal to improve a certain control target and realize the tracking of the desired trajectory. Reference [17] used high-speed camera to carry out clinical gait experimental analysis and obtained the expected joint motion curves of hip joint and knee joint of lower limb exoskeleton robot in sagittal plane as shown in Figure 3. Through the cftool data fitting toolbox of MATLAB 2014b, the expected motion trajectory of the lower limb exoskeleton robot in the hip joint and knee joint is defined as

$$
\begin{gathered}
p_{\text {hip }}(x)=21.39 \sin (4.27 x-0.58)+8.92, \\
p_{\text {knee }}(x)=43.14 \sin (2.74 x-0.65)+31.4 \sin (4.8 x+8.91),
\end{gathered}
$$

where $p_{\text {hip }}(x)$ and $p_{\text {knee }}(x)$ denote the expected motion angle of hip joint and knee joint, respectively, and $X$ represents the exercise time. The expected joint trajectory of lower limb exoskeleton robot in sagittal plane is shown in Figure 3.

3.1. Basic Principles of Iterative Learning Control. In this control technology, iterative learning always starts from an initial point, and almost all convergence proofs require the same initial conditions [18]. When designing an iterative learning system, in order to ensure the convergence of the system, the initial value of the iteration at the beginning of each iteration should be consistent with the expected initial value [19]:

$$
y_{i}(0)=y_{k}(0) \quad i=0,1, \ldots k,
$$

where $y_{i}(0)$ represents the initial value of iteration and $y_{k}(0)$ is the expected initial value. At the beginning of learning, the initial state of the system is $y_{0}(0)$. The $\lambda_{k+1}(x)$ motion error $\varepsilon_{k+1}(x)$ is reduced by learning the control law. It is shown in the structure diagram of D-type learning law system. Its control method is as follows: the $K+1$ control $\lambda_{k+1}(x)$ is equal to the correction term of the $k$ th control $\lambda_{k}(x)$ plus the output error $\varepsilon_{k}(x)$ of the $k$ th time [13], and the iterative learning control law is

$$
\lambda_{k+1}(x)=\lambda_{k}(x)+\mu_{k}(x) \Gamma \varepsilon_{k}(x),
$$

where $\lambda_{k+1}(x)$ denotes the $K+1$ control, $t \in[0, t], K$ represents the number of iterations, $\mu_{k}(x)>1$, and $\Gamma$ represents the learning gain coefficient.

The complete closed-loop control system includes control modules, execution modules, feedback modules, and controlled objects. The specific process is to use the PC

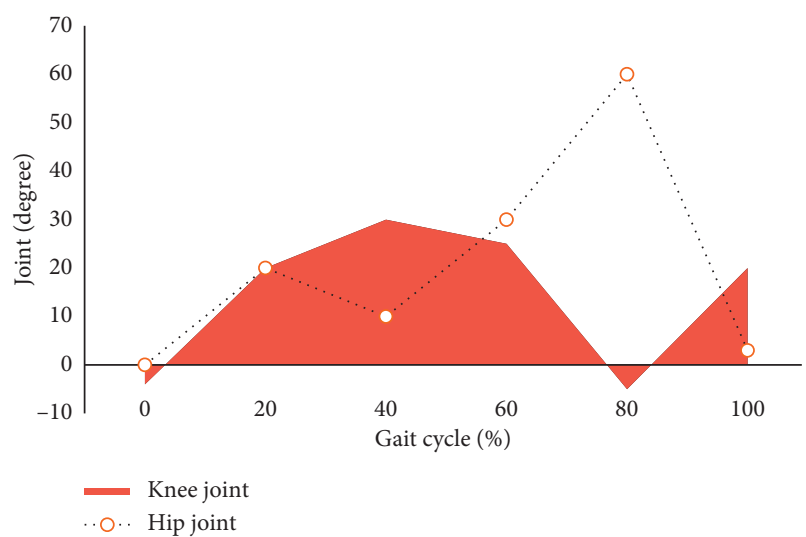

FIgURE 3: Expected joint trajectory of lower limb exoskeleton robot in sagittal plane.

console as the control module to output control commands to the neuromuscular electrical stimulator as the execution module through serial communication. The electrical stimulator generates electrical stimulation pulse sequences that act on the target muscles through stimulation electrodes, while the feedback module uses angles [20]. The sensor collects the knee joint angle and feeds it back to the console in real time. The following will briefly introduce the characteristics and functions of each part of the hardware system. The PC console is a hardware platform that implements control algorithms and delivers control instructions to the electrical stimulator. This research is based on the framework and uses $\mathrm{C}++$ language to write software programs to realize the control algorithm. The software program can realize the functions of real-time calculation of the required control amount, data communication with the electrical stimulator, and control interface display [21]. The calculation of the control quantity is to obtain the error information by comparing the actual angle obtained by the angle sensor with the expected angle and calculate the current required control quantity based on the control algorithm designed above; the data communication module is responsible for obtaining the actual angle from the angle sensor in real time and outputting the control amount to the electric stimulator in real time; the control interface display is used for the operator to observe the control effect in real time and evaluate and adjust. The main hardware system required by the system is shown in Figure 4 .

In order for robots to serve humans, the medium of information exchange between humans and machines is indispensable. The motion control function is the core of the lower limb exoskeleton rehabilitation robot to achieve different strategies for the rehabilitation mode [22]. Therefore, the quality of the motion control directly determines whether the robot can complete tasks efficiently and accurately, as well as the safety and comfort of the user. The lower limb exoskeleton rehabilitation robot is in direct contact with the user, and its failure may cause the robot to be destroyed and the user to suffer serious injury. Therefore, besides realizing basic motion control, its safety protection function is also very important. Based on the basic 


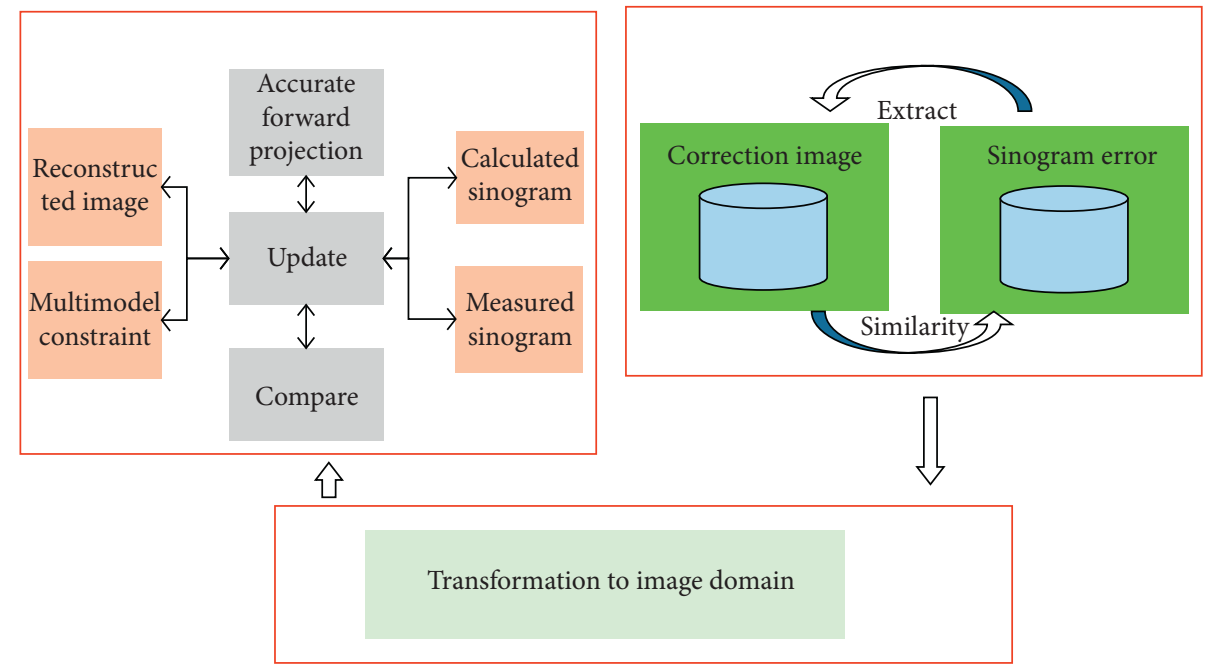

FIgURE 4: D learning law system structure.

requirements of the above functions, the control system of the lower limb exoskeleton rehabilitation robot is designed.

3.2. Design of Motion Control System. The control task of the lower limb exoskeleton robot is to track the desired trajectory of repeated gait. After each iteration cycle, the lower limb exoskeleton robot returns to its original position. By learning the control law, $\lambda_{k}(x)$ is designed to reduce the $k$ th motion error [23].

The convergence of the learning efficiency is determined by the spectral radius of the control law, that is, the convergence of the learning efficiency is inversely proportional to the spectral radius, and the necessary and sufficient condition for the convergence of the exponential variable gain closed-loop D-type iterative learning control rate is the spectral radius $\lambda<1$.

The spectral radius of closed-loop D-type controller is shown in Figure 5, where the learning gain coefficients are 50,100 , and 120, respectively. It can be seen from Figure 5 that the spectral radius of the learning gain coefficient of 100 is lower than that of the other two gain coefficients, and the convergence speed is faster. It can be clearly seen from Figure 5 that when the learning gain coefficient in the exponential variable gain closed-loop D-type iterative learning control rate is 100 , after one cycle of iterative learning, the spectral radius decreases rapidly, and the corresponding convergence speed increases.

From the third iterative learning cycle, the spectral radius reaches the minimum, and the corresponding convergence speed reaches the fastest, and the spectral radius is in the whole range. During the movement, it is assumed that the speed is a constant, and the convergence of motion control system is improved with the increase of movement time. On the basis of exponential variable gain closed-loop D-type iterative learning control rate, the spectral radius which affects the convergence rate of motion control system decreases with the increase of time $t$, and the convergence rate of iterative learning is inversely proportional to the

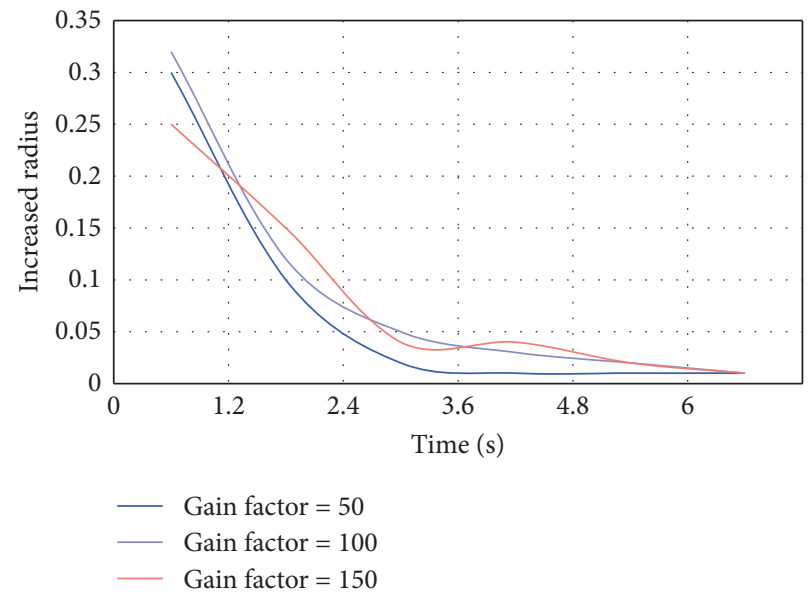

Figure 5: Spectral radius of closed-loop controller.

spectral radius. Therefore, the convergence speed of iterative learning increases gradually. From the third iteration cycle, the spectral radius is the smallest and the convergence speed is the fastest, which realizes the integration of the humancomputer system.

\section{Analysis of Simulation Results}

4.1. The Proposed Control Targets for Stormwater Runoff Control in Central Urban Areas. The goal of lower limb exoskeleton rehabilitation robot is to replace rehabilitation doctors to assist patients with gait rehabilitation training and improve the efficiency of rehabilitation training and the effect of rehabilitation treatment. After three-dimensional modeling, theoretical calculation, simulation analysis, processing, and assembly, the physical prototype of the lower limb exoskeleton rehabilitation robot is basically completed. This section mainly introduces the construction of the system experimental platform, the hardware integration and debugging in the control system, and the design of the human-computer interaction interface of the rehabilitation 
training mode selection module in the software system [16]. After the completion of the system, a series of experiments including functional test, passive rehabilitation training, and active auxiliary training were carried out. The experiment not only verifies the feasibility of the structure and the effectiveness of the algorithm but also provides a theoretical basis for further proposing more ideal ontology structure and control strategy.

The exoskeleton must be wearable, so the bone shape and the distribution of degrees of freedom must be studied before the configuration design. Through the study of the mechanism of human lower limb bones, the lower limb bones are composed of thigh bones, calf bones, and foot bones, which are, respectively, formed by three parts of skeletal joints, knee joints, and step joints, and the entire lower limbs are formed by two series mechanisms in parallel [17]. In order to study the movement of the human body, ergonomics and clinical medicine usually use the sagittal plane, frontal plane, and horizontal plane to describe. In terms of the structural form of the joints, each joint movement is not strictly about the center of the sphere but is a compound movement of sliding and rotating. However, considering the relatively small amount of sliding, it is generally treated as an ideal spherical pair. The spherical rotation of the joint can be decomposed into rotation around three two-by-two orthogonal joint axes, which are abduction/adduction, extension/flexion, and internal rotation/ external rotation, as shown in Table 1 . Due to the limitation of ligament length, the amplitudes of the rotation angles of the skeleton joint, knee joint, and step joint in the three directions are quite different. The skeletal joint has 3 degrees of freedom, which can realize abduction/adduction, extension/flexion, and internal rotation/external rotation; the knee joint has 1 degree of freedom, which can only achieve extension/flexion movements; the stepping joint has 3 degrees of freedom, which can achieve abduction/adduction, toe flexion/dorsiflexion, and internal rotation/external rotation movement. The range of rotation angle of each joint of the lower limbs can be obtained from the statistical results of ergonomics.

The lower extremity exoskeleton rehabilitation robot studied in this subject is a device used for gait rehabilitation training for patients. The gait is mainly composed of the flexion/extension movement of each joint in the sagittal plane, so the movement of other planes is not studied. The lower extremity exoskeleton can be simplified into a 7-bar model with 6 degrees of freedom, that is, the left and right leg joints, knee joints, and step joints each have 1 degree of freedom of rotational movement, and the patient's balance is maintained by a weight-reducing balance mechanism.

In addition, we take the joint movement angles of the exoskeleton orthosis shown in Table 2 as the design index of the lower extremity exoskeleton orthosis.

Because the musculoskeletal system of different people shows great differences, it is necessary to identify the parameters of the musculoskeletal system of different subjects before the formal experiment. The iteration speed of the
TABLE 1: Range of rotation angle of each joint of lower limbs.

\begin{tabular}{lccc}
\hline Joint & $\begin{array}{c}\text { Extension/ } \\
\text { bending }\end{array}$ & $\begin{array}{c}\text { Abduction/ } \\
\text { adduction }\end{array}$ & $\begin{array}{c}\text { Internal rotation/ } \\
\text { external rotation }\end{array}$ \\
\hline $\begin{array}{l}\text { Hip joint } \\
\text { Knee }\end{array}$ & $145 / 25$ & $35 / 25$ & $55 / 55$ \\
joint & $0 / 135$ & 0 & 0 \\
$\begin{array}{l}\text { Ankle } \\
\text { joint }\end{array}$ & $34 / 25$ & $20 / 20$ & $25 / 20$ \\
\hline
\end{tabular}

TABLE 2: Joint motion range of lower limb exoskeleton orthosis.

\begin{tabular}{lccc}
\hline Joint & Movement & $\begin{array}{c}\text { Standard gait } \\
\text { angle }\end{array}$ & $\begin{array}{c}\text { Exoskeleton } \\
\text { design angle }\end{array}$ \\
\hline $\begin{array}{l}\text { Hip flexion/extension } \\
\text { exercise }\end{array}$ & $150 / 235$ & $25 / 15$ & $35 / 20$ \\
$\begin{array}{l}\text { Knee joint flexion/ } \\
\text { extension exercise }\end{array}$ & $0 / 130$ & $0 / 55$ & $0 / 60$ \\
$\begin{array}{l}\text { Ankle joint flexion/ } \\
\text { extension exercise }\end{array}$ & $30 / 20$ & $25 / 30$ & $25 / 15$ \\
\hline
\end{tabular}

model-based ILC algorithm proposed in this paper is related to the accuracy of the model, so good model parameters can further accelerate the iterative learning process when the iteration speed is already fast. The parameters that need to be identified include human inertia parameters, joint motion parameters, and muscle activation and contraction parameters. This article looks for subjects to participate in human joint motion control experiments. Some subjects cannot get rid of their own random control and other influencing factors, so follow-up experiments are not ideal. Based on the data of human body size in adult body size and the relative mass distribution of human body segments, the main sizes and masses of lower limbs are obtained as shown in Table 3.

Everyone's height and size are different. If we want to ensure that the lower limb exoskeleton robot can meet the physiological characteristics of human body, we must fully consider this point in the design. According to the probability distribution research of ergonomics measurement, it is concluded that there is a direct proportional relationship between the size of the lower limb bone and the height of Chinese adults. According to the established motion control equation of the lower limb exoskeleton robot, considering the external interference, the expected trajectory of the lower limb exoskeleton robot hip joint and knee joint and the actual tracking trajectory based on iterative learning control are shown in Figure 6. Due to the influence of the robot load disturbance, it acts on the exponential gain closed-loop D-type iterative learning control rate [18]. Then, according to the error performance function between the expected value and the actual value of the joint, the weight is modified iteratively to make the actual tracking of the hip joint and knee joint closer to the expected trajectory.

The change of gait trajectory tracking error in the iterative learning control process is shown in Figure 7. By comparing and analyzing the spectral radius of the closed- 
TABle 3: Size and mass of lower limbs.

\begin{tabular}{lcc}
\hline Body segment name & Mass $(\mathrm{kg})$ & Length $(\mathrm{mm})$ \\
\hline Thigh & 8.247 & 542.39 \\
Lower leg & 2.942 & 436.92 \\
\hline
\end{tabular}

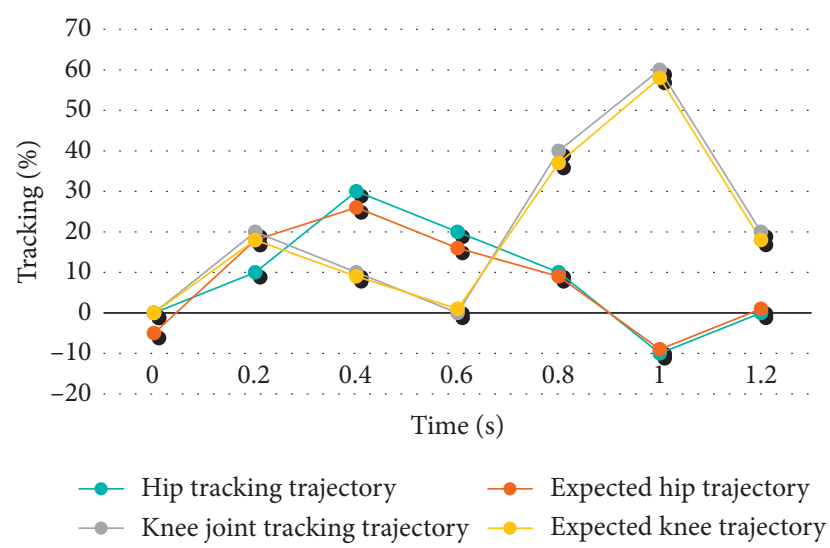

FIGURE 6: Trajectory tracking results based on iterative learning control.

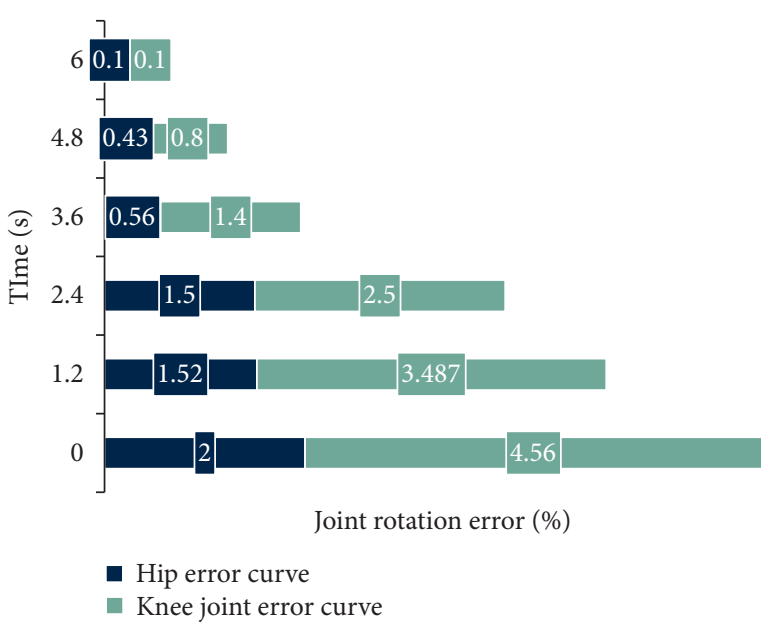

FIGURE 7: The change of gait trajectory tracking error in iterative learning control process.

loop D-type controller shown in Figure 5, the two have the same conclusion, that is, in the first two iteration cycles, the spectral radius of the exponential gain closed-loop D-type iterative learning control rate decreases rapidly, the corresponding convergence speed increases, and the trajectory tracking error of the lower limb exoskeleton robot is gradually reduced. When the spectral radius reaches the minimum, the corresponding convergence speed reaches the fastest and the smallest trajectory tracking error is obtained [19], so that the lower limb exoskeleton robot can move with the human body quickly and accurately. However, the initial tracking error of hip joint and knee joint is large because the lower limb exoskeleton robot needs response time to move with the human body [24].

\section{Conclusion}

The joint motion control system is designed based on the ILC algorithm, and the simulation analysis is carried out. Aiming at the problem of slow iteration speed and insufficient accuracy of the traditional ILC algorithm in the control system, the musculoskeletal model was introduced, the ILC electrical simulation algorithm based on the musculoskeletal model was designed, and its control effect was verified through simulation analysis. The joint motion control experiment research was launched, including the complete design of the joint motion experiment program, the parameter identification of the musculoskeletal system of different subjects, and the development and result analysis of joint motion control experiments. It is verified that the ILC electrical simulation algorithm based on the musculoskeletal model has a good control effect in human joint motion control.

Aiming at the problem of providing good posture balance assistance in the rehabilitation process of lower limb paralysis patients, taking the standing posture balance as an example, the human posture balance experimental research has been carried out, including the design of experimental schemes, the development of experiments, and the analysis of experimental results. Finally, the law of human body posture balance and the mechanism of lower limb joints in the process of maintaining posture balance of the human body are discussed, which has positive significance for the subsequent design of a lower limb motor function rehabilitation system that integrates the ability of posture balance.

In the walking posture of human body with repetitive motion, the motion control model of lower limb exoskeleton robot is established based on iterative learning control algorithm. The exponential gain closed-loop D-type motion control system is designed by MATLAB software, and the expected trajectory of human lower limb hip joint and knee joint is obtained. The simulation results show that due to the following response time in the initial stage, there is a large error in the initial stage of the lower limb exoskeleton robot following the human body. After two iterations, the expected trajectory tracking error of the hip joint and knee joint is basically eliminated, which effectively solves the errors of the human body's hip and knee joints' expected trajectory tracking error and poor servo performance.

\section{Data Availability}

The data used to support the findings of this study are available from the corresponding author upon request.

\section{Conflicts of Interest}

The authors declare that they have no conflicts of interest.

\section{References}

[1] Q. Zhang, Y. Meng, L. Wu, X. Xiang, and C. Xiong, “Artificially induced joint movement control with musculoskeletal 
model-integrated iterative learning algorithm," Biomedical Signal Processing and Control, vol. 5, no. 9, pp. 101-108, 2020.

[2] B. Ren, X. Luo, and J. Chen, "Single leg gait tracking of lower limb exoskeleton based on adaptive iterative learning control," Applied Sciences, vol. 9, no. 11, pp. 2251-2252, 2019.

[3] B. Huo, Y. Liu, Y. Qin, B. Chu, and C. T. Freeman, "Disturbance observer based iterative learning control for upper limb rehabilitation," InIECON 2020 The 46th Annual Conference of the IEEE Industrial Electronics Society, vol. 1, no. 8, pp. 2774-2779, 2020.

[4] L. Wu, Q. Wu, Q. Zhang, and C. Xiong, "Electrically induced joint movement control with iterative learning algorithm," International Conference on Advanced Robotics and Mechatronics (ICARM), vol. 2, no. 7, pp. 426-430, 2017.

[5] Z. Sun, F. Li, G. Wang, Y. Liu, Y. Lian, and K. Liu, "A novel RBF neural network-based iterative learning control for lower limb rehabilitation robot with strong robustness," Chinese Control Conference (CCC), vol. 7, no. 1, pp. 4454-4459, 2019.

[6] Z. Sun, F. Li, Y. Lian, S. Liu, and K. Liu, "An adaptive iterative learning control approach for lower limb rehabilitation robot in noisy environments," IEEE 9th Annual International Conference on CYBER Technology in Automation, Control, and Intelligent Systems (CYBER), vol. 2, no. 9, pp. 905-910, 2019.

[7] Q. Miao, H. S. Lo, S. Q. Xie, and H. S. Li, "Iterative learning control method for improving the effectiveness of upper limb rehabilitation," 23rd International Conference on Mechatronics and Machine Vision in Practice (M2VIP), vol. 1, no. 2, pp. 1-5, 2016.

[8] M. Ketelhut, F. Göll, B. Braunstein, K. Albracht, and D. Abel, "Iterative learning control of an industrial robot for neuromuscular training," IEEE Conference on Control Technology and Applications (CCTA), vol. 8, no. 1, pp. 926-932, 2019.

[9] V. Ghanbari, V. H. Duenas, P. J. Antsaklis, and W. E. Dixon, "Passivity-based iterative learning control for cycling induced by functional electrical stimulation with electric motor assistance," IEEE Transactions on Control Systems Technology, vol. 27, no. 5, pp. 2287-2294, 2018.

[10] S. Liu, D. Meng, L. Cheng, and M. Chen, "An iterative learning controller for a cable-driven hand rehabilitation robot," InIECON 2017-43rd Annual Conference of the IEEE Industrial Electronics Society, vol. 10, no. 1, pp. 5701-5706, 2017.

[11] K. Yin, M. Pang, K. Xiang, J. Chen, and S. Zhou, "Fuzzy iterative learning control strategy for powered ankle prosthesis," International Journal of Intelligent Robotics and Applications, vol. 2, no. 1, pp. 122-131, 2018.

[12] P. Müller, C. Balligand, T. Seel, and T. Schauer, "Iterative learning control and system identification of the antagonistic knee muscle complex during gait using functional electrical stimulation," IFAC-PapersOnLine, vol. 50, no. 1 , pp. 8786-8791, 2017.

[13] Y. Li, A. An, J. Wang, and H. Zhang, "Iterative learning control of exponential variable gain based on initial state learning for upper limb rehabilitation robot," Chinese Automation Congress (CAC), vol. 11, no. 22, pp. 4947-4952, 2019.

[14] J. Realmuto, G. Klute, and S. Devasia, "Preliminary investigation of symmetry learning control for powered ankle-foot prostheses," Wearable Robotics Association Conference (WearRAcon), vol. 2, no. 5, pp. 40-45, 2019.

[15] F. J. Abu-Dakka, A. Valera, J. A. Escalera, M. Abderrahim, A. Page, and V. Mata, "Passive exercise adaptation for ankle rehabilitation based on learning control framework," Sensors, vol. 20, no. 21, pp. 6215-6216, 2020.
[16] W. Ting and S. Aiguo, "An adaptive iterative learning based impedance control for robot-aided upper-limb passive rehabilitation," Frontiers in Robotics and AI, vol. 7, no. 4, pp. 41-42, 2019.

[17] D. Ke, Q. Ai, W. Meng, C. Zhang, and Q. Liu, "Fuzzy PD-type iterative learning control of a single pneumatic muscle actuator," Intelligent Robotics and Applications, vol. 7, no. 16, pp. 812-822, 2017.

[18] G. Sebastian, Z. Li, Y. Tan, and D. Oetomo, "On implementation of feedback-based PD-type iterative learning control for robotic manipulators with hard input constraints," IEEE 15th International Conference on Control and Automation (ICCA), vol. 7, no. 16, pp. 43-48, 2019.

[19] D. Shen and X. Li, "A survey on iterative learning control with randomly varying trial lengths: model, synthesis, and convergence analysis," Annual Reviews in Control, vol. 48, no. 8, pp. 89-102, 2019.

[20] Z. Li, W. Chen, J. Zhang, and S. Bai, "Design and control of a 4-DOF cable-driven arm rehabilitation robot (CARR-4). In2017," IEEE International Conference on Cybernetics and Intelligent Systems (CIS) and IEEE Conference on Robotics, Automation and Mechatronics (RAM), vol. 11, no. 19, pp. 581-586, 2017.

[21] V. Molazadeh, Z. Sheng, X. Bao, and N. Sharma, "A robust iterative learning switching controller for following virtual constraints: application to a hybrid neuroprosthesis," IFACPapersOnLine, vol. 51, no. 34, pp. 28-33, 2019.

[22] J. Xia, D. Huang, Y. Li, and N. Qin, "Iterative learning of human partner's desired trajectory for proactive human-robot collaboration," International Journal of Intelligent Robotics and Applications, vol. 4, no. 2, pp. 229-242, 2020.

[23] T. Seel, C. Werner, and T. Schauer, "The adaptive drop foot stimulator - multivariable learning control of foot pitch and roll motion in paretic gait," Medical Engineering \& Physics, vol. 38, no. 11, pp. 1205-1213, 2016.

[24] Y. Sun, J. Xu, H. Wu, G. Lin, and S. Mumtazau, "Deep learning based semi-supervised control for vertical security of maglev vehicle with guaranteed bounded airgap," IEEE Transactions on Intelligent Transportation Systems, vol. 1, 2021. 